\title{
La formación de profesoras y profesores en didáctica de la historia. Valoraciones en torno a qué historia enseñar para la formación ciudadana"
}

\author{
The training of teachers in didactics of history. \\ Assessments around what history to teach for citizen training \\ Miguel Ángel Jara ${ }^{a}$ \& María Celeste Cerdé ${ }^{b}$ \\ aUniversidad Nacional del Comahue, Neuquén, Argentina.凶 mianjara@gmail.com \\ [orcid.org/0000-0002-0959-9000] \\ bUniversidad Nacional de Córdoba, Córdoba, Argentina. celepater@gmail.com \\ [orcid.org/0000-0002-4429-2821]
}

\section{RESUMEN}

La Didáctica de la Historia se ha constituido en un espacio de disputas y de sentidos sobre los contenidos de la historia investigada y la historia enseñada ante los desafíos de la formación ciudadana en el siglo XXI. En Argentina la historia escolar ha ido lentamente revisando sus contenidos para atender a la diversidad de situaciones y fenómenos que acontecen en este mundo. Sobre la base de lo que piensa un grupo de estudiantes del profesorado en historia de dos Universidades Nacionales en Argentina, ofrecemos un análisis de los aspectos comunes sobre las finalidades, los contenidos y los problemas sociales que el estudiantado nos ha proporcionado a partir de la utilización de un cuestionario. Se trata de una investigación educativa cualitativa crítica que tiene como objetivo conocer las representaciones de estudiantes en formación para ofrecer oportunidades epistemológicas y didácticas que aventuren mayores posibilidades en la enseñanza de la historia escolar a partir de problemas sociales relevantes y candentes.

PALABRAS CLAVE: didáctica de la historia, formación ciudadana, historia, problemas sociales.

\section{ABSTRACT}

The Didactics of History has become a space for disputes and meanings about the contents of the investigated history and the history taught in the face of the challenges of

Proyecto de Investigación C129 "El aprendizaje escolar de las ciencias sociales en contextos de la cultura digital" (2017-2020), financiado y asentado en la Facultad de Ciencias de la Educación de la Universidad Nacional del Comahue-Argentina. 
civic education in the century. XXI. In Argentina, school history has been slowly revising its contents to attend to the diversity of situations and phenomena that occur in this world. Based on what a group of students of the history faculty at two National Universities in Argentina think, we offer an analysis of the common aspects of the purposes, contents and social problems that the student body has provided us from the use of a questionnaire. This is a critical qualitative educational research that aims to know the representations of students in training to offer epistemological and didactic opportunities that venture greater possibilities in the teaching of school history from relevant and burning social problems.

KEY WORDS: didactics of history, citizen training, history, social problems.

"La ciudadanía democrática debería ser, sin lugar a dudas, la meta principal de una enseñanza que ha de poner el énfasis en lo que nos hace humanos, en todos los derechos de todas las personas y en la dignidad humana por encima de cualquier otra cosa. No hay absolutamente nada que justifique las diferencias económicas, sociales, políticas, culturales, religiosas, etc. y mucho menos las injusticias” (Pagès 2019, p. 25).

\section{LOS CONTEXTOS DE LA FORMACIÓN DEL PROFESORADO EN HISTORIA}

En Argentina de las 57 Universidades Nacionales Públicas, más de 20 forman a profesoras y profesores en historia, sin contar las Universidades Privadas y los Institutos de Formación Docentes, en algunas provincias del territorio. Dos de ellas, la Universidad Nacional del Comahue (UNCo) ubicada en la norpatagonia argentina y la Universidad Nacional de Córdoba (UNC) en el centro del país, tienen la carrera de profesorado en historia. La UNCo a poco de cumplir 50 años como institución formadora ofrece, en la Facultad de Humanidades, una carrera con un plan de estudios organizado en 29 asignaturas, en cinco años de duración. Las 3 áreas de conocimiento histórico (General, Latino Americana y Argentina) y el área Teórica (Metodología, Historiografía, Política y Economía) totalizan 24 asignaturas a las que se les suma, en el quinto año, 4 asignaturas didácticas/pedagógicas y un idioma extranjero. El plan de estudios, con algunas modificaciones, está vigente desde el año 1986 y, como se puede inferir de la organización curricular, posee una importante formación disciplinar frente a una escasa formación pedagógica y didáctica para un perfil de profesorado. Los cuatro primeros años son compartidos con la Licenciatura en Historia, trayecto que se separa en el quinto año según sea para el profesorado o la licenciatura.

La UNC, conocida como la Docta, fue una de las primeras Universidades Públicas de Argentina, fundada en el año 1613. Desde el año 1957, en la Facultad de Filosofía y Humanidades, se forma profesoras y profesores en historia. El plan de estudios vigente desde el año 1993, se ha modificado atendiendo a los criterios y estándares nacionales -algo que la UNCo aún no ha realizado-. Se organiza en un ciclo introductorio común (licenciatura y profesorado), un ciclo de formación y un ciclo de formación profesional. Sólo nos detendremos en caracterizar el plan de estudios correspondiente al profesorado. En el primero y segundo ciclo encontramos 13 asignaturas disciplinares o afines, un taller de aplicación, 4 espacios 
denominados conexos y 2 asignaturas optativas ${ }^{1}$, un idioma extranjero y 3 asignaturas pedagógicas/didácticas, todas distribuidas de primero a cuarto año. El Ciclo de Formación Profesional, ubicado en el quinto año del trayecto, se conforma con dos espacios, el taller de la práctica docente y residencia (anual) y un espacio conexo. Se trata de un plan de estudios de 25 asignaturas en el que, si bien las asignaturas denominadas pedagógicas no se encuentran todas juntas en el quinto año como lo es para el caso de la UNCo, comparte una misma dificultad: la escasa formación pedagógica y didáctica para un perfil de profesorado.

En este marco, nuestra indagación situada, pretende realizar un análisis atendiendo a los aspectos comunes relacionados a la Didáctica de la Historia, no es nuestra intención establecer comparaciones, ni generalizaciones, sino dar cuenta de los problemas comunes con los que nos encontramos en un campo de conocimiento específico, con la finalidad de ofrecer oportunidades situadas e innovadoras en la formación inicial del profesorado en historia. El origen de las preocupaciones comunes se encuentra en la pertenencia a la Asociación de Profesoras/es de Enseñanza de la Historia en Universidades Nacionales (APEHUN), que no sólo nos ha permitido encontrarnos en espacios/redes para compartir experiencias e investigaciones, sino, fundamentalmente, establecer lazos de trabajo solidarios y colaborativos entre cátedras de diferentes Universidades Públicas Nacionales de Argentina.

Sabemos que formar para la enseñanza de un conocimiento particular como lo es la historia, requiere pensar profundamente no sólo en los aspectos referidos a un objeto que en sí mismo es inacabado, sino también en cómo ese objeto se aprende a enseñar en contextos y situaciones singulares. Es aquí donde la didáctica cumple un rol central en la formación del profesorado. Joan Pagès sostiene que:

La didáctica de la historia pretende dar respuesta a esta formación. Se ocupa de enseñar el oficio de enseñar historia, de formar como profesores a los estudiantes de historia. Pero, ¿en qué consiste preparar para ser profesor o profesora de historia?, ¿qué significa formar las competencias en didáctica de la historia de los futuros profesores? En mi opinión, preparar a un profesor o una profesora de historia, enseñar a enseñar historia, consiste en educar a un profesional para que tome decisiones, sepa organizarlas y llevarlas a la práctica, sobre los conocimientos históricos que debe enseñar en un aula de un centro determinado. Aprenda que enseñar historia en secundaria consiste en preparar a los jóvenes para que se sitúen en su mundo, sepan interpretarlo desde su historicidad y quieran intervenir en él con conocimiento de causa, quieran ser protagonistas del devenir histórico (Pagès, 2004, p. 158).

Desde la perspectiva del didactista catalán la formación del profesorado debería atender a la articulación entre el conocimiento histórico, las prácticas situadas y las finalidades de la historia escolar para la formación de ciudadanías. Se trata de unos principios que configuran la construcción teórica de un campo de conocimiento que, entre

1 Materias conexas de cursado obligatorio: Antropología social y cultural, Sociología, Teoría política y Economía política. Se ofrece diversidad de asignaturas optativas en el ámbito de la escuela de historia, la Facultad y la Universidad. 
otras cuestiones, pone el acento en la reflexión epistemológica del conocimiento histórico y los usos públicos de ese conocimiento con la intención de que contribuya a la comprensión del mundo, en el devenir de una múltiple temporalidad, para la intervención democrática en la construcción de futuros.

La educación histórica en la escuela siempre ha tenido como finalidad formar ciudadanías y, como también sabemos, esta formación ha estado definida -no sin disputas y tensiones- desde las políticas públicas dominantes en cada época. De acuerdo a los proyectos educativos, al menos en los últimos cincuenta años en Argentina, identificamos dos proyectos claramente antagónicos, los que promueven una ciudadanía pasiva, receptora y reproductora de valores y conocimientos hegemónicos y los que procuran formar para el desarrollo de la participación democrática de las jóvenes generaciones, cuestionadoras de un status quo dominante.

Se trata de una situación que también se presenta como una cuestión que preocupa en otros ámbitos. Por ejemplo, Ramón López Facal (2000) reflexiona en torno a la realidad española y plantea que el profesorado que no se cuestiona la utilidad social de la profesión, se encuentra en la disyuntiva de orientar la enseñanza de acuerdo con los criterios dominantes, inadecuados y hasta contradictorios en cuanto a promover una mayor democratización de la sociedad. Para el autor esto se refleja en la mayoría de las disposiciones oficiales (decreto de mínimos) y en la mayoría de los manuales o textos escolares; o, por el contrario, asumir valores contrahegemónicos tratando de sustituir la competitividad individualista por la cooperación, la exaltación satisfecha de la propia identidad por el respeto a la diversidad y el pluralismo, o la sacralización del progreso técnico alcanzado por las sociedades occidentales por la solidaridad activa con las personas excluidas del bienestar. Dice López Facal que la enseñanza de la historia y las ciencias sociales debe contribuir a desarrollar, en el estudiantado, las capacidades críticas que posibiliten reflexionar sobre el mundo que nos rodea y los problemas más acuciantes de nuestra sociedad, superando la mera transmisión de informaciones (acríticas). Desde esta perspectiva, la capacidad crítica implica poder realizar análisis de complejidad creciente y, además, ser capaz de formular propuestas alternativas a las carencias y deficiencias de aquello que se rechaza. En definitiva, se trata de formar una ciudadanía crítica que rechace modelos sociales basados en la desigualdad y la exclusión y construya una práctica política de transformación de la sociedad con criterios de mayor equidad y solidaridad (López Facal, 2000).

La persistencia de ciertas tradiciones indica que hay que repensar la formación del profesorado para que, como sostiene Pagès (2009) en la cita anterior, pueda tomar decisiones, sepa organizarlas y llevarlas a la práctica. En este marco resulta relevante saber qué conocimiento histórico se ofrece en la formación del profesorado, para poder identificar los procesos de desarrollo de un pensamiento didáctico de la historia que contribuya a la formación de ciudadanías.

Este escrito, con intenciones de develar qué conocimientos históricos se aprenden para enseñar, lo organizamos sobre la base de la pregunta inicial atendiendo a dos momentos estrechamente relacionados, los aportes de la historiografía y las representaciones, perspectivas y finalidades que el estudiantado en formación considera son claves para la 
formación ciudadana. Son datos e información que obtuvimos de un cuestionario ${ }^{2}$ que se organizó en tres grandes apartados. En el primero indagamos aspectos generales sobre el estudiantado que nos permitió tener un primer dato sobre edad, trayectoria formativa, área de interés en la formación del profesorado, valoraciones sobre la enseñanza y el aprendizaje, entre otros. El segundo apartado, un conjunto de preguntas semiestructuradas, con opciones abiertas y argumentaciones breves, indagó sobre aspectos específicos a la asignatura Didáctica de la Historia en el marco de la formación y del plan de estudios. Finalmente, el tercer apartado -que es el que mayoritariamente tomamos para el análisis en este escritoestuvo organizado por cinco preguntas, con el mismo criterio del apartado anterior, centradas fundamentalmente en las finalidades de la Didáctica de la Historia articulando la historia investigada y la historia enseñada desde las perspectivas, valoraciones y representaciones del estudiantado encuestado en dos Universidades Nacionales de Argentina ${ }^{3}$.

El esquema general de la presentación analítica de los datos e información, en este escrito, parte de las representaciones, perspectivas y valoraciones que el estudiantado encuestado nos no proporcionó y pone en diálogo, no lineal ni excluyente, el pensamiento de estudiantes en formación, el contexto y las finalidades, todo en relación con la pregunta que nos orienta ¿Qué historia enseñar para la formación ciudadana? Y lo presentamos de la siguiente manera (Tabla 1).

\section{¿QUÉ HISTORIA ENSEÑAR PARA LA FORMACIÓN CIUDADANA? LOS APORTES DE LAS DISCIPLINAS EN LA FORMACIÓN INICIAL DEL PROFESORADO}

En este trabajo describimos y analizamos la información y los datos obtenidos a partir de la utilización de un cuestionario que han completado estudiantes de ambos profesorados que cursan la asignatura Didáctica de la Historia en el año 20204. El cuestionario nos permitió

2 El instrumento ha sido utilizado en diversas investigaciones a nivel nacional e internacional, con adaptaciones y ajustes a los requerimientos de cada investigación y contexto. Surge de las primeras investigaciones del GREDICS de la UAB, ha sido utilizado en las investigaciones doctorales de la autora y autor de este artículo. Se trata de un instrumento que, evidentemente, es efectivo para la consecución de los procesos investigativos, validado en diversas pesquisas. Por ejemplo, en los artículos citados de Jara et al. (2016a) o el de González-Valencia et al. (2019) recuperan el cuestionario, su estructura y organización para dialogar con los datos obtenidos en cada investigación.

3 El cuestionario en investigaciones educativas cualitativas críticas y con el método de estudio de caso (Stake, 2005), se ha convertido en el campo de la investigación en didáctica de las ciencias sociales en un instrumento potente que, junto a otros (entrevistas en profundidad, grupo focal, análisis documental, relatos y narrativas, etc.), permite construir conocimiento situado. Para el caso concreto de la investigación que aquí presentamos -parcialmente- el criterio estuvo orientado por investigaciones anteriores y, fundamentalmente, por la idea de profundizar en la dimensión específica de las finalidades en la formación didáctica del profesorado en historia. Como en las investigaciones referenciadas, la población con la que hemos trabajado el cuestionario es la que anualmente cursa la asignatura, por lo tanto, no aleatoria ni representativa, de allí que, como estudio de caso, es intrínseca a nuestra preocupación. El cuestionario ha sido trabajado con el estudiantado a través de la plataforma google drive, de manera virtual durante la última semana de marzo de 2020, previo al inicio del cursado de la asignatura.

4 En este proceso indagatorio, en el marco de la pandemia COVID 19 y ante la imposibilidad de un cursado de la asignatura de manera presencial, hemos decidido realizar el cuestionario de manera virtual, utilizando la herramienta que ofrece el google drive, con un óptimo resultado. 
Tabla 1. Esquema análisis de la formación, el contexto y las finalidades, desde el pensamiento del estudiantado.

\begin{tabular}{cc}
\hline \multicolumn{2}{c}{ Pensamiento del Estudiantado } \\
\hline de la historia & de la ciudadanía \\
\hline Formación Situada del Profesorado \\
\hline Hinalidaderia Investigada Enseñada \\
\hline ¿Para qué enseñar?
\end{tabular}

Fuente: Elaboración propia.

tener una primera aproximación a las valoraciones y representaciones que el estudiantado tiene sobre la Didáctica de la Historia, en especial aquellos componentes relacionados con las finalidades, el contenido, las estrategias, los materiales didácticos y las expectativas, entre otros. En el análisis pondremos énfasis en aquellos componentes que nos posibiliten dar cuenta de la pregunta inicial ¿Qué historia enseñar para la formación ciudadana?, sin descuidar la estrecha relación con el espacio específico de formación, las finalidades de la enseñanza y los contenidos que el estudiantado manifiesta son fundamentales para contribuir a la formación de la ciudadanía.

El cuestionario fue completado por 77 estudiantes que, mayoritariamente, tienen entre 21 y 30 años de edad. En el trayecto de la formación del profesorado el estudiantado encuestado se concentra en los últimos tres años de la carrera, el 46,8\% cursa el cuarto año, el 35,1\% quinto año y el $18,2 \%$ tercer año ${ }^{5}$. Se trata de un grupo de estudiantes que manifiesta que su área de interés por conocimiento histórico es variada en temas, escalas y períodos de los procesos históricos ${ }^{6}$. Por ejemplo, el área de Historia Contemporánea (26), de Historia Argentina (17), Historia de América Latina (15), Historia Social, Política y Cultural (13), otras -Antigua y Media(6). Mayoritariamente se inclinan por los procesos históricos de los siglos XX y XXI a escala nacional, regional y mundial. Para el caso de la Historia Contemporánea los procesos de Asia, África y Europa han sido señalados como relevantes en el interés del estudiantado.

La pregunta 6 del cuestionario, relacionada con la anterior, requería que nombraran las tres asignaturas que les ha ofrecido la posibilidad de pensar en la enseñanza. Si bien las respuestas han sido muy diversas -en cuanto a los espacios curriculares cursados- igual que en los datos anteriores se mantuvo una tendencia dentro de las áreas en las que reagrupamos la información obtenida en la pregunta 5 del cuestionario. Por ejemplo, el área de Historia Contemporánea (39), de Historia Argentina (41), Historia de América Latina (27), Historia Social, Política y Cultural (8) e Historia Antigua y Media (20). En este caso se incorpora el Área Teórica (15), el Área pedagógica y didáctica (25) y asignaturas optativas, como Geografía (6), entre otras. ¿De qué manera han contribuido a pensar en la enseñanza? El estudiantado

Que las y los estudiantes puedan cursar Didáctica de la Historia a partir del segundo año de la formación se debe al régimen de correlatividades que no impide que así sea.

6 Ante la diversidad de temas históricos que interesan al estudiantado y debido a la falta de precisión en la ubicación de áreas de conocimiento historiográfico, hemos agrupado las respuestas, de la pregunta número 5, atendiendo a una clasificación según se organiza el plan de estudios de los profesorados. 
argumenta, igualmente, desde diferentes experiencias la reconstrucción subjetiva de ellas. Para poder reflejar los sentidos de las argumentaciones del estudiantado diremos que el cursado de las diversas asignaturas del plan de estudios, hasta antes de llegar a la Didáctica de la Historia, ha contribuido a pensar en la enseñanza a partir de:

a) Las formas metodológicas utilizadas por las profesoras o profesores de las cátedras. Esta categoría incluye, atendiendo al orden de importancia que le asigna el estudiantado, a las estrategias de participación y desarrollo del contenido (exposiciones, debates, resolución de actividades, presentación de temas utilizando dispositivos digitales), los materiales y recursos didácticos utilizados (videos, fuentes, bibliografía) y al tratamiento de conceptos para la comprensión de procesos históricos (otredad, poder, subjetividades, etc.); a modo de graficar las argumentaciones una encuestada nos dice:

"En primera instancia, la organización de la cátedra materializada en las aulas virtuales y programas de las asignaturas son muy buenas. En segundo lugar, la disposición para con los estudiantes a la hora de proporcionar material extra, consultas, etc. En tercer lugar, la forma de dar clases, los tonos utilizados, las consignas, las propuestas desarrolladas en clase. En cuarto lugar la coherencia entre lo dictado y lo evaluado, lo pedido y lo efectivamente corregido en las diferentes instancias de evaluación. Por último, en las tres asignaturas logré llegar a cuestionarme en numerosas ocasiones la forma en la que los conocimientos me fueron enseñados durante mi paso por el nivel primario y el nivel medio. Desde las metodologías y debates propuestos hasta las posibilidades de buscar nuevas perspectivas sobre acontecimientos y procesos que pensábamos que conocíamos muy bien” (C. 62).

b) La reflexión epistemológica promovida por las profesoras o los profesores de las cátedras. Categoría que refiere a los procesos de tensión, cuestionamiento y modos de producción del conocimiento histórico, en el que se ha procurado pensar en otros relatos, visibilizar a otras y otros, construir otras periodizaciones para dar cuenta de temporalidades múltiples y diversas. Un estudiante plantea que:

"Estas materias me han llevado a pensar de qué manera transmitir los contenidos de manera que generen en los alumnos un pensamiento crítico. Haciendo referencia principalmente a aquellos sectores de la historia que, según he visto desde mi propio aprendizaje, han sido omitidos o nombrados al pasar, dejando fuera su participación en la Historia. También intentando lograr que el alumno logre ser consciente de lo que es la Historia Oficial, y se plantee una mirada crítica, que lo lleve a ir más allá de esa historia de grandes hombres (C. 12).

c) Las relaciones entre historia, enseñanza y escuela en las explicaciones de las profesoras o profesores de las cátedras. Incluye la diversidad de aspectos que son objeto de la Didáctica de la Historia. 
"Estas tres materias me han permitido ampliar mis horizontes del conocimiento histórico, y al hacerlo me han generado ciertas concepciones respecto a la naturaleza del conocimiento y cómo debe ser impartido. El cómo está delimitado, el currículum de las materias históricas mencionadas así como fueron enseñadas por los profesores me han permitido pensar respecto a la naturaleza de la enseñanza. La enseñanza es la transmisión de conocimientos, estas materias así como otras me han transmitido conocimientos pero cada una de ellas lo ha transmitido de una forma específica en base al currículo propuesto. Esto me permite pensar sobre las distintas formas de transmisión de conocimientos en base a las propias características de los contenidos propuestos" (C. 45).

Identificar el área de conocimiento histórico, como las asignaturas del profesorado que les han posibilitado pensar en la enseñanza son datos relevantes para nuestra investigación porque, como luego veremos, son indicios en los que se configuran pensamientos y sentidos sobre la historia que desean enseñar. Entre las asignaturas de interés y las asignaturas que han ofrecido la posibilidad de pensar en la enseñanza se destacan la Historia Contemporánea para el ámbito nacional, latinoamericano y europeo. En el próximo apartado presentamos los datos referidos a los contenidos que el estudiantado manifiesta no debería dejar de enseñarse en la clase de historia de la escuela media.

\section{¿QUÉ HISTORIA ENSEÑAR PARA LA FORMACIÓN CIUDADANA? REPRESENTACIONES, PERSPECTIVAS Y FINALIDADES DEL ESTUDIANTADO EN FORMACIÓN}

Son abundantes y diversas las producciones en el campo de la didáctica de la historia que se han ocupado de indagar en las Representaciones Sociales (RS), en las Perspectivas Prácticas (PP) y en las finalidades de la enseñanza, influenciadas por los aportes teóricos de Moscovici (1979), Jodelet (1986), Adler (1984) Addler y Adler (1994), , entre otros. En el ámbito iberoamericano, por nombrar sólo algunas de las más recientes, las investigaciones de Pagès (1996, 1997, 2007, 2009), Jara y colaboradores (2010, 2012, 2016a, 2016b), Santisteban (2011), Cerdá (2018), González-Valencia y colaboradores (2020) indican que tanto las RS y las PP son claves para comprender las subjetividades construidas por el estudiantado y, atendiendo al planteo de Pagès (1996), se trata de trabajar con y en ellas, no contra ellas, para producir cambios en relación a concepciones sobre la historia, la enseñanza y el aprendizaje.

El estudiantado encuestado conjuga experiencias diversas, inscritas en un contexto cambiante, vertiginoso e incierto, característica propia de esta época. Son jóvenes que manifiestan estar en un profesorado en historia, por diversas razones. En una de las preguntas del cuestionario, indicamos que señale una de las cinco opciones que les ofrecimos. El ítem indicaba: si una persona les pregunta ¿por qué estudias historia? ¿Qué le dirías? Mayoritariamente señala como respuesta que le gusta la historia (39\%). Un 26\% indica le gusta enseñar historia, un 22\% dice que estudiar historia nos permite saber sobre nuestro pasado, un 1,3\% manifiesta que es una salida laboral rápida. El 11,7\% restante marcó la opción otra, 
pero no indicó cuál. Los datos son relevantes en el sentido de que prevalece el gusto o interés por la historia o el conocimiento histórico frente a la idea de pensarlo como objeto de enseñanza o como una finalidad. En su conjunto, el $88,3 \%$ de los datos, son indicios de que el interés, la enseñanza y las finalidades son RS y PP que configuran una cierta relación entre para qué y qué historia enseñar.

En cuanto a si consideran que las personas que tendrán como estudiantes en la escuela secundaria tienen las mismas posibilidades de aprendizaje, le hemos solicitado que señalen el enunciado con el que más acuerdan, según reproducimos en la Tabla 2.

Tabla 2. Valoraciones sobre las posibilidades de aprendizaje escolar.

\begin{tabular}{lc}
\hline Opciones con las que acuerdan & Rtas. \\
\hline a) Sí, tienen las mismas posibilidades de aprender todo. & 6 \\
\hline b) Sí, después de un tiempo de estar escolarizados/as en la cultura histórica. & 0 \\
\hline c) Sí, si prestan atención al profesor/a y cumplen con las tareas. & 2 \\
\hline d) No, nunca pueden tener las mismas posibilidades porque son diferentes. & 20 \\
\hline $\begin{array}{l}\text { e) No, pero sí pueden aprender si el profesor/a les ofrece conocimientos significativos para } \\
\text { sus vidas. }\end{array}$ & 49 \\
\hline
\end{tabular}

Fuente: Elaboración propia.

Como puede observarse en los datos, mayoritariamente, el estudiantado parte de la postura de que no todas las personas que tendrán como estudiantes tienen las mismas posibilidades de aprender historia. Los ítems d y e representan el 89,6\% de acuerdos. Si bien, de este total de acuerdos, un $26 \%$ indica que la imposibilidad radica en que las y los jóvenes y adolescentes son diferentes, es importante que el 63,6\% restante del estudiantado considera que habrá posibilidades de que las personas aprendan historia en la escuela secundaria en la medida que se les ofrezcan conocimientos significativos. Subyace en esta idea una finalidad, política y epistemológica, que atribuye a la historia escolar una función clave para comprender nuestro presente y los que nos pasa. El futuro profesorado asigna a la historia un rol protagónico para la vida, en la medida que sea significativo el conocimiento histórico que se enseñe. Aspecto que refuerza la idea de que la historia tiene mucho para ofrecer a la formación de ciudadanas y ciudadanos del siglo XXI, si pensamos que mayoritariamente la historia contemporánea es el área de interés del estudiantado encuestado.

En el orden de lo conocido, también sabemos que la educación es una práctica eminentemente política y que desde el área de las ciencias sociales, hoy, resulta poco viable pensar en enseñanzas neutrales y menos aún desprovistas de perspectivas teóricas o reflexiones epistemológicas. Esta cuestión, en la historia investigada y la historia enseñada es una obviedad, aunque no desconocemos que persisten tradiciones o posicionamientos que confunden adoctrinamiento con formar para la autonomía, con conocimientos que permitan argumentar las decisiones que configuran acciones o intervenciones en la sociedad. Se trata de posturas ideológicas que se disputan los sentidos por la historia y qué ciudadanía formar.

En este sentido, en el apartado tres del cuestionario, que se organizó en torno de las finalidades en la enseñanza, hemos preguntado al estudiantado lo siguiente: "El hecho de que un profesor/a se plantee una perspectiva ideológica para la enseñanza de la historia, 
consideras que es...". La escala de valoraciones ofrecidas va de muy negativo a muy positivo. Los datos obtenidos son: muy negativo 1,3\%; negativo 13\%; ni negativo ni positivo $37,7 \%$; positivo $37,7 \%$ y muy positivo $10,4 \%$. Los datos pueden ofrecernos diversidad de interpretaciones, pero si los consideramos en el marco de las finalidades en la enseñanza el panorama se nos ajusta a una articulación de dos agrupamientos de las valoraciones. En primer lugar que el 37,7\% considera que es ni negativo ni positivo e igual porcentaje que considera que es positivo. En segundo lugar, si consideramos la totalidad de negativo y muy negativo $(14,3 \%)$ es claramente inferior a las valoraciones positivas y muy positivas $(48,1 \%)$. De este agrupamiento surge una preocupación a partir del dato, no menor, que indica que al 37,7\% del estudiantado le parece indistinto el posicionamiento ideológico en la enseñanza de la historia.

El papel de la ideología en la enseñanza, el tema de la neutralidad y los valores, entre otras dimensiones, resultan fundamentales dado que intervienen en la configuración de los propósitos que las profesoras y los profesores otorgan a sus prácticas de enseñanza. Las dificultades con respecto a las cuestiones de "neutralidad", "objetividad" pueden estar vinculadas no sólo a posicionamientos personales de los profesoras/es y/o futuras profesoras/es, sino también a culturas o tradiciones escolares que entendían a la escuela como un "espacio neutral".

En el cono sur, las indagaciones sobre esta problemática se encuentran básicamente vinculadas a la enseñanza de la Historia Reciente. Los estudios de Toledo Jofré (2015) muestran que el 65\% del profesorado asume una estrategia de posicionamiento "neutralidad activa” al momento de la enseñanza de la dictadura en Chile. En Argentina, Jara (2012), en un estudio sobre estudiantes y profesores en su primer año de ejercicio docente, también reconoce la influencia de las ideas de objetividad y subjetividad en el carácter conflictivo que éstos le asignan a la enseñanza de la Historia Reciente. González (2014a) reconoce, por un lado, la presencia de cierta ilusión de neutralidad relacionada con una concepción de profesionalidad que demanda, desde otros actores escolares, criterios de "objetividad" e "imparcialidad", pero advierte, también, la existencia de otro tipo de posicionamiento de involucramiento y beligerancia. Una posición similar -la del "profesor militante"- describe Funes y colaboradores (2007) en sus estudios en la Patagonia argentina. Queda pendiente el interrogante acerca de si la neutralidad o beligerancia -retomando las propuestas de Funes son maneras de ser/pensar o de actuar en función de determinados momentos, en un contexto también particular, como lo es el ámbito educativo y, no menor, repensar la cuestión ética en la enseñanza.

\section{¿QUÉ HISTORIA ENSEÑAR PARA LA FORMACIÓN CIUDADANA? LOS CONTENIDOS ENTRE TEMAS/PROBLEMAS DOMINANTES, EMERGENTES, LATENTES Y RESIDUALES}

En el apartado 3.4 del cuestionario, preguntábamos: ¿Qué temas/problemas te parecen no deberían dejar de enseñarse en la clase de historia en la escuela media? Escríbelos en orden de importancia. Para dar cuenta de la diversidad de respuestas del estudiantado, hemos construido cuatro categorías, que consecuentes con el proceso 
metodológico adoptado, en el que los estudios de casos considerados en contexto (Stake, 2005), siguiendo las consideraciones de Campbell y Stanley (1963) (citado en Stake, 2005), nos permiten en rigor contar con una base empírica para falsear generalizaciones. Esta estrategia metodológica nos permite, en tanto base empírica, la generación de conceptos y teoría convirtiéndola en un elemento esencial para la investigación social. En este sentido la teoría fundamentada contribuye y es oportuna a nuestra investigación. Como sostienen Strauss y Corbin (2002) en la Teoría Fundamentada es importante la recopilación sistemática de datos y el análisis, porque la teoría que surja de ello guarda estrecha relación entre sí, los autores sostienen que:

"Un investigador no inicia un proyecto con una teoría preconcebida (a menos que su propósito sea elaborar y ampliar una teoría existente). Más bien comienza con un área de estudio y permite que la teoría emerja a partir de los datos. Lo más probable es que la teoría derivada de los datos se parezca más a la "realidad" que la teoría derivada de unir una serie de conceptos basados en experiencias o sólo especulando. Debido a que las teorías fundamentadas se basan en datos, es más posible que generen conocimientos, aumenten la comprensión y proporcionen una guía significativa para la acción (Strauss \& Corbin, 2002, pp. 13-14).

Desde esta perspectivita, entonces, las categorías que hemos creado dialogan entre la información ofrecida por el estudiantado, los planes de estudio y las escuelas historiográficas que son objetos de estudio en los dos profesorados en cuestión. El agrupamiento que sigue nos permite realizar el proceso analítico, recuperando algunos ejemplos en la Tabla 3.

Tabla 3. Temas/Problemas que no deberían dejar de enseñarse en la clase de historia.

3.4. ¿Qué temas/problemas te parecen no deberían dejar de enseñarse en la clase de historia en la escuela media. Escríbelos en orden de importancia

\begin{tabular}{|c|c|c|}
\hline & Categorías & Número de respuestas \\
\hline \multirow[b]{2}{*}{ a) } & \multirow{2}{*}{$\begin{array}{l}\text { Historia centrada en la historiografía como fuente para } \\
\text { enunciar contenidos escolares. }\end{array}$} & $\begin{array}{c}\text { Agenda tradicional } \\
\text { (16) }\end{array}$ \\
\hline & & $\begin{array}{l}\text { Agenda renovada } \\
\text { (19) }\end{array}$ \\
\hline b) & $\begin{array}{l}\text { Historia centrada en problemas sociales como fuentes para } \\
\text { enunciar contenidos escolares. }\end{array}$ & (17) \\
\hline c) & $\begin{array}{l}\text { Historia centrada en referentes temporales como fuentes } \\
\text { para enunciar contenidos escolares. }\end{array}$ & (10) \\
\hline \multirow{2}{*}{ d) } & \multirow{2}{*}{$\begin{array}{l}\text { Historia centrada en componentes de la realidad social } \\
\text { como fuentes para enunciar contenidos escolares }\end{array}$} & $\begin{array}{l}\text { Tradicionales } \\
\quad(5)\end{array}$ \\
\hline & & $\begin{array}{l}\text { Renovados } \\
\text { (1) }\end{array}$ \\
\hline e) & $\begin{array}{l}\text { Historia centrada en las finalidades como fuentes para } \\
\text { enunciar contenidos escolares. }\end{array}$ & (9) \\
\hline
\end{tabular}




\section{Historia centrada en la historiografía como fuente para enunciar contenidos escolares}

En esta categoría se incluye enunciaciones pertenecientes a las narrativas historiográficas, en las que podemos diferenciar las perspectivas clásicas con aquellas nuevas perspectivas que se han instalado, recientemente, en la agenda y reflejan los avances y las innovaciones teóricas en el campo. En un primer subgrupo, podemos identificar a aquellas respuestas en que prima una enunciación de las tradicionales "edades", organizadas y cristalizadas en programas de estudios universitarios (43\%). Una perspectiva en que "toda" la historia debe/ merece ser enseñada:

Historia Argentina-historia de Europa-Prehistoria (3);

Historia general. Historia reciente. Historia de culturas diferentes. Prehistoria;

Historia Argentina, Historia Latinoamericana, Historia Contemporánea;

Historia Contemporánea de Argentina (especialmente las partes más conflictivas y controversiales, que son muy poco abordadas, o que se abordan polémicamente. El mejor ejemplo son los golpes de Estado, no vistos en muchas escuelas);

Historia Contemporánea de América (haciendo hincapié en la historia caribeña, casi no vista, especialmente procesos revolucionarios como el cubano de la década del ‘50);

Historia Moderna de América y Europa (tratando la violenta interacción entre ambos continentes de la manera menos conflictiva);

Historia Antigua y Medieval (ya que, aun siendo muy lejana a nuestro continente, ha sido determinante en la formación de nuestra cultura y hay que seguir enseñando los procesos de esos milenios);

Me gustaría añadir que se debería dar también la Historia de Asia, África y Oceanía, para al menos salir del secundario con una visión generalizada de lo que ocurrió en esas partes del mundo, que si bien nos son lejanas, tienen una influencia determinante en el mundo actual que, globalizado como está, nos impactan los sucesos que ocurren en esas partes del planeta.

En un segundo subgrupo dentro de esta categoría, incluimos aquellas respuestas (66\% del total) que recuperan la nueva agenda historiográfica, pero mantienen los saberes académicos -al menos en su enunciación- como principal criterio de referencia al momento de seleccionar contenidos para la enseñanza:

Los problemas de género, la cuestión de los derechos humanos, la historia local y nacional (como la formación del Estado argentino), la historia de los sectores populares, indígenas, trabajadores.

1) Historia de los sectores populares, las mujeres y todo tipo de minorías (étnicas, sexuales, religiosas, etc.) 2) Dictaduras, observar lo que sucedió en el pasado así no se repite en el presente o el futuro 3) Historia Latinoamericana 4) Historia local 5) Historia no occidental/europea; otros tipos de cultura. 


\section{Historia centrada en problemas sociales como fuentes para enunciar contenidos escolares}

En esta categorización entendemos a los problemas sociales no como una dificultad, sino como una oportunidad de reflexión epistemológica y cognitiva de situaciones, fenómenos o acontecimientos que pueden ser abordados, dada su complejidad, desde perspectivas del campo de las ciencias sociales. Son cuestiones socialmente vivas, candentes o conflictivas y generan controversia en su tratamiento. Se trata de problemas que pueden señalarse como relevantes por su grado de significatividad para la comprensión de los procesos históricos. En este apartado, las respuestas son variadas (22\% del total), pero podemos reconocer algunas recurrencias en cuanto a las cuestiones de género, DD.HH., diversidad y desigualdad. Algunos ejemplos:

La desigualdad, pensada no sólo en término de clases, sino también de género, para poder pensar en conjunto con los estudiantes el sistema en el que vivimos.

Derechos humanos, historia reciente, procesos históricos regionales, globales y cómo afectan a nuestro contexto. Pueblos originarios, reconocer cómo fueron actuando los distintos grupos de poder a lo largo de nuestra historia.

Diversidad/ Identidad de género; Violencia de género; Feminismo; Movimientos Sociales actuales; Ley de Educación Sexual (no se implementa en la mayoría de las escuelas y si lo hacen es de manera superficial).

\section{Historia centrada en referentes temporales como} fuentes para enunciar contenidos escolares

Esta categoría refiere a las dimensiones u operadores de la temporalidad que posibilitan la comprensión de los procesos históricos. Si bien el tiempo histórico es un componente constitutivo base de las operaciones historiográficas y un criterio de periodización en la selección y organización de contenidos, escasamente se constituyen en contenidos específicos en la enseñanza de la historia. No obstante ello, en algunas de las respuestas (13\% del total) podemos encontrar nociones temporales tales como cambio y continuidad, causalidad, la historia como proceso, la noción de cambio social y las relaciones pasado-presente. Algunas de las respuestas que ubicamos en este grupo:

En mi opinión, esos temas serían: primeramente, la importancia del conocimiento histórico como disciplina y como desarrollador de una mentalidad crítica; los principales períodos históricos, vistos en líneas generales pero rescatando las continuidades y rupturas de cada uno; a su vez, indagar sobre los principales aportes que cada uno de ellos contribuye para el desarrollo de la humanidad como se la conoce hoy en día; prestando especial atención a todos los agentes que conjugan en los procesos históricos en cuestión (C. 23). 
Lo que puedo ofrecer es una serie de temas/problemas que considero que deberían enseñarse, dado que no sé qué es en realidad lo que se enseña actualmente en general y, por tanto, no puedo decir qué de ello no quisiera que deje de enseñarse. Hecha esa aclaración, pienso que debería enseñarse: la conflictividad social como motor del cambio histórico; la multicausalidad y la multidimensionalidad de los fenómenos y procesos históricos; la noción de que somos un producto histórico no acabado, sino en constante devenir (C. 7).

La importancia de los procesos. Concepto como genocidio. Enseñar otras culturas y dejar un poco el eurocentrismo. Profundizar aún más sobre historia argentina (C. 29).

Laincidencia del pasado sobre el presente, la capacidad colectiva de transformación de la realidad histórica, protagonismo popular en la historia, mujeres, y disidencias en la historia, historia política nacional, crítica al capitalismo (C. 68).

En consonancia con los resultados de las investigaciones, el futuro no resulta un constructo temporal incorporado por los y las futuros/as profesores, a excepción de sólo una respuesta de las 77 que conforman la muestra:

1. dilema de la identidad 2. derechos humanos y lugar de la memoria 3. la interpretación de pasado, la percepción del presente y las expectativas del futuro (C. 47).

\section{Historia centrada en componentes de la realidad social como fuentes para enunciar contenidos escolares}

En este caso, referimos a los componentes o dimensiones de la trama social que posibilita focalizar en una de ellas para enseñar historia. Las dimensiones tradicionales en el campo refieren a la social, la política, la económica, la cultural, entre otras y escasamente se mencionan al componente de la crisis, el conflicto, la diversidad o la multiculturalidad, por ejemplo, como dimensiones constitutivas de la complejidad social. Relacionada en gran parte a las formas particulares en que las perspectivas estructurales de la historia ingresan y se cristalizan como una especie de sobre entendido teórico-práctico. Las divisiones de la realidad histórica en dimensiones forman parte de una de las tradiciones más extendidas en la selección y organización de contenidos de la historia escolar (6\% del total).

Lo social, económico y político.

La temática social y cultural.

Pasado reciente. Dictadura y democracia. La inserción de Argentina en el mundo.

Historia social, política y económica de pueblos, campesinos y comunidades indígenas.

En muy pocos casos, esa realidad social/histórica es caracterizada a partir de otros elementos, como es el caso del conflicto ( $2 \%$ del total): 
Económicos/desigualdades sociales o de clase. Racismo, diversidades/ creo que son conflictos históricos y actuales.

Conflictos sociales, multicausalidad de los procesos, continuidades históricas.

\section{Historia centrada en las finalidades como fuentes para enunciar contenidos escolares}

La categoría refiere a una clara indistinción entre el para qué o el porqué y el qué de la enseñanza. En este caso reconocemos finalidades relacionadas concretamente con el objeto de la historia y aquellas enunciaciones muy generales que nos son privativas de la historia. En este segundo caso, se trata de enunciaciones enmarcadas en decisiones políticas, ideológicas, cognitivas y utilitarias (12\% del total).

Desnaturalizar el orden de las cosas; 2) Comprender su origen; 3) Proyectar un futuro deseable (C. 15).

Desarrollo de los valores de la humanidad. Aprender la historicidad del presente. Desarrollar conciencia histórica y pensamiento histórico crítico (C. 34).

El pensamiento crítico en cualquiera de sus medidas, pero creo que eso es lo primordial, el criticarse el orden establecido, la cultura imperante, el sistema, la hegemonía en cualquier ámbito o espacio creo que es fundamental, básicamente porque viene de la mano con un saber histórico, viene de la mano con procesos o desarrollos de la humanidad o de las sociedades que se gestan en una etapa de la historia y que al día de hoy repercuten. Y por otro lado también que no se pierda de vista nunca los conflictos, ya sean todas las oposiciones, las resistencias, las diferencias o divergencias ya que eso también es el motor de la humanidad. Y por último la comprensión del "otro", ya que en historia constantemente estamos hablando de un "otro" en otro contexto espacio-temporal (C 52).

De este análisis inicial de las respuestas de los y las estudiantes surgen algunas cuestiones interesantes de compartir, que nos gustaría enunciar en términos de tensiones/ desafíos en tanto muestran la existencia de diversas tradiciones o perspectivas acerca de la selección de contenidos para la historia escolar. En efecto, formas tradicionales de responder a este interrogante coexisten con propuestas renovadas al momento de responder "qué" contenidos deben tener presencia en las aulas. En términos generales podemos advertir la presencia de tipos de respuestas que, a los fines analíticos, hemos sistematizado en la Fig. 1.

En el conjunto de las respuestas, existe una preeminencia del modelo tradicional (que hemos caracterizado como mundo-burbuja, siguiendo los aportes de García Pérez, F., 2016) frente a las tendencias más renovadas que proponen las cuestiones socialmente relevantes como una alternativa posible/deseable al código tradicional de la enseñanza de la historia y sus insuficiencias para la comprensión/transformación de la realidad social e histórica. Pero, entre ambas opciones surge un conjunto de respuestas que incorporan algunas de las preocupaciones/propuestas del campo de la Didáctica de la Historia, como por ejemplo, la enseñanza de la temporalidad, en menor medida visiones complejas de 


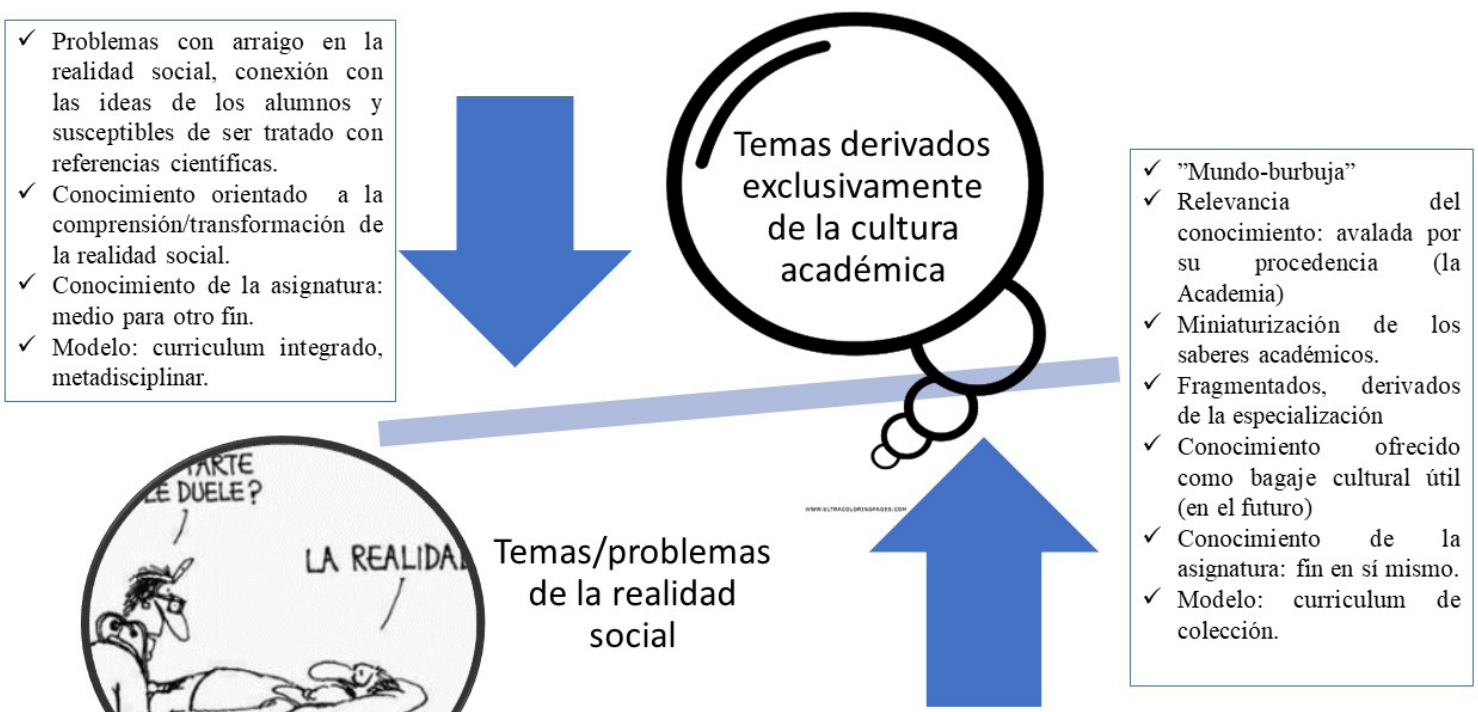

Fig. 1. Temas/problemas de la Realidad Social.

Fuente: Elaboración propia.

la realidad social (conflictiva, diversa, no accesible a "primera vista"). También, podemos reconocer como positivo la importante presencia de contenidos que -con Paula González (2018)- denominaremos emergentes y latentes (Fig. 2):

A partir del análisis de las respuestas de las y los estudiantes, podemos reconocer la preeminencia de respuestas que pueden ser ubicadas en las categorías de contenidos

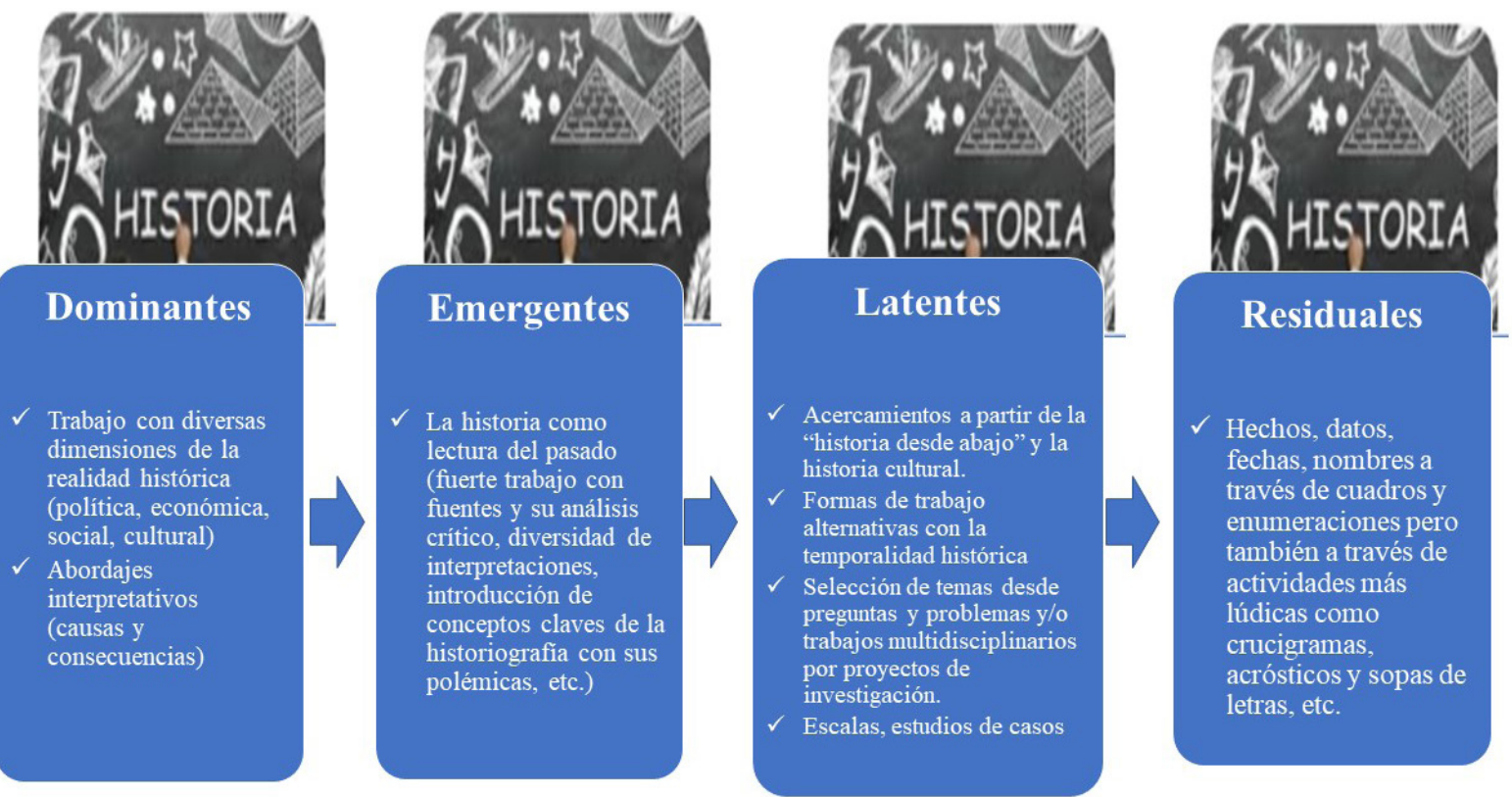

Fig. 2. Historias: Dominantes, Emergentes, Latentes y Residuales.

Fuente: Adaptación de información extraída de González (2018). Elaboración propia. 
"emergentes" o "latentes", por sobre aquellos que han caracterizado los contenidos/temas/ problemas de la enseñanza de la historia tanto en su versión tradicional, pero también distanciándose, en parte, de algunos de los que se establecieron luego de la reforma de los años noventa en el sistema educativo argentino.

En cuanto a los contenidos "dominantes", si bien aparecen como parte de las respuestas enunciados tales como "lo político, lo económico, lo social”, no resultan cuantitativamente relevantes; con igual presencia encontramos abordajes interpretativos, al menos de la enunciación de aquello que el estudiantado considera no debería dejar de enseñarse en la clase de historia. En menor medida, aún aparecen algunos contenidos "residuales"; cuando lo hacen se encuentran vinculados a la historia nacional o bien a enunciaciones de ciertos relatos que han invisibilizado a otras y otros protagonistas de los procesos históricos.

Algunos temas/problemas de la temporalidad, la incorporación de perspectivas de los sectores subalternos, de género, la diversidad, entre otros, son algunos de los contenidos renovados que tienen una importante presencia en las respuestas obtenidas. Cuestión que aventura otros modos de pensar en otras finalidades en la enseñanza de la historia, aspectos relevantes y necesarios para profundizar en un contexto de inmediatez, incertidumbres y cambios culturales profundos en nuestras sociedades.

Además de estas "presencias", podríamos reconocer también ciertos temas escasamente manifiestos en las representaciones de los futuros profesores y profesoras: la historia como forma de conocimiento, los juegos de escala que permiten romper con el corsé del EstadoNación e incorporar, por ejemplo, la escala global/local, la enseñanza de temporalidades alternativas, los estudios de caso como formas alternativas a las perspectivas holísticas que priman en la enseñanza de la historia.

Finalmente es notable el número de respuestas que relacionan contenidos de la historia con finalidades de su enseñanza. La investigación ha demostrado como fundamental los propósitos o finalidades que las y los docentes le atribuyen a la enseñanza de su asignatura. Esta relevancia obedece a distintos motivos, pero, principalmente, se asienta en el consenso existente sobre el impacto que los propósitos tienen en las decisiones que toman los profesores en su práctica y en la necesidad de su incorporación -como objeto de identificación y reflexión- tanto en los dispositivos de formación inicial como en el desarrollo profesional (Evans, 1991; Thorton, 1994; Pagès, 1994, 2011; Barton \& Levstik, 2004; Van Hover \& Yeager, 2007, entre otros).

Autores como Barton y Levstik (2004) señalan que las concepciones de las y los profesores sobre las finalidades tienen un mayor impacto en la práctica docente que sus concepciones sobre el contenido del conocimiento de la asignatura y las estrategias que utilizan para su enseñanza. Evans (1991), por su parte, afirma que la concepción sobre los objetivos es un factor determinante para el significado que los docentes le atribuyen a la enseñanza de la Historia. Finalmente, también Pagès y Santisteban reafirman este supuesto, al sostener que las finalidades influyen en todos los aspectos del currículum: en la selección de los contenidos, en las estrategias de enseñanza y aprendizaje, los materiales curriculares, las actividades o evaluación. De una u otra forma están presentes en todas las actividades educativas, ya sea de manera explícita o como currículum oculto (2011). 


\section{A. Jara \& M. C. Cerdá}

Así las cosas, las respuestas que los futuros profesores y profesoras -aun con la indistinción señalada entre finalidades/temas-problemas- pueden señalar también una confusión deseable según la cual la dimensión cognitiva de la historia (aquello que conoce y la forma en que lo conoce) difícilmente puede separarse de sus potencialidades identitarias, políticas, etc.

\section{UN CIERRE INCONCLUSO, PARA SEGUIR PENSANDO EN LA FORMACIÓN DEL PROFESORADO Y LA EDUCACIÓN CIUDADANA}

Decíamos anteriormente que en este escrito nos proponíamos develar qué conocimiento histórico se ofrece en la formación del profesorado, para poder identificar los procesos de desarrollo de un pensamiento didáctico de la historia que contribuya a la formación de ciudadanías. En el derrotero analítico nos encontramos con la diversidad y heterogeneidad de perspectivas, finalidades y sentidos de los "usos públicos" del conocimiento histórico. También de ciertas regularidades que han configurado tradiciones en las prácticas de educación histórica que, al menos desde las intenciones, aventuran posibilidades que ameritan profundizar la reflexión teórica y metodológica.

La persistencia de ciertas tradiciones coexiste con nuevas aproximaciones, más colindantes a finalidades de la enseñanza de la historia orientadas al desarrollo del pensamiento histórico y la formación de ciudadanía crítica y participativa. Así, podemos reconocer:

- narrativas de neutralidad sobre la enseñanza de la historia/reconocimiento de la politicidad de la finalidad y saberes históricos

- relevancia de los contenidos derivada exclusivamente de su procedencia académica/relevancia de los contenidos en función de su aporte a la comprensión de problemas socialmente relevantes.

- perspectivas holistas, estructuralistas, de grandes síntesis/historia desde abajo, escalas diversas.

- historia como relato del pasado/ historia como forma de conocimiento.

El estudio preliminar que hemos realizado nos ha ofrecido un caudal de información y datos inconmensurable para pensarnos en la formación y en la enseñanza de la historia en consonancia con la formación de ciudadanías plurales, diversas, críticas y participativas para fortalecer y construir permanentemente sociedades democráticas con justicia social. Quizás el mayor desafío sea pasar de la enunciación/intención/diagnóstico a la acción/ práctica/innovación ${ }^{7}$ de una historia escolar que vuelva a contribuir a repensarnos humanos. El profesorado necesita repensarse, en este siglo XXI, con nuevas perspectivas; pero sin desconocer aquello que es valioso para la configuración de un pensamiento didáctico de la historia que se presente como síntesis de opciones corrosiva de lo dado (Jara, 2017).

7 En próximos escritos pondremos en diálogo los resultados obtenidos de los cuestionarios con las propuestas didácticas elaboradas por las y los estudiantes. 
Iniciamos este escrito con una cita de Joan Pagès, que forma parte de una reflexión profunda sobre ¿qué profesorado, qué ciudadanía, qué futuro? que ha realizado en el marco de su clase magistral en la Universidad Autónoma de Barcelona en el año 2018, con motivos de acogerse a los beneficios de la jubilación. En el sentido de las preguntas que expresan preocupación/desafío/enseñanza/futuro planteadas por Joan Pagès, consideramos que estas primeras indagaciones, aquí compartidas, intentan aportar desde el campo de la Didáctica de la Historia, conocimiento acerca de algunas cuestiones que consideramos centrales para repensar los dispositivos de formación de futuros profesores. Un debate necesario y urgente para otra historia posible en este complejo siglo XXI.

\section{REFERENCIAS}

Adler, P. (1984). A Field Study of Selected Student Teacher Perspectives toward Social Studies. Theory and Research in Social Education, XII(1), 13-30.

Adler, P. A., \& Adler, P. (1994). Observational Techniques. En N. K. Denzin \& Y. S. Lincoln (Eds.), Handbook of Qualitative Research (377-392). London: Ed. Sage.

Barton, K., \& Levstik, L. (2004). Teaching History for the Common Good. New Jersey: Mahwah. Lawrence Erlbaum Associates, Inc.

Evans, R. (1991). Concepciones del maestro sobre la Historia. En Boletín de Didáctica de las Ciencias Sociales, 1, 3-4.

Funes, G. A., Ertola, F. M., \& Zorzini, A. P. (2007). La temporalidad en la enseñanza de lo reciente/presente. Avances de una indagación en los 5tos años de las escuelas medias de San Carlos de Bariloche. Revista Espacio regional, 2(4), 37-46.

García Pérez, F. F. (2016). Educar en la escuela para afrontar los problemas del mundo. En Más allá de lo imposible. La dimensión política de los derechos humanos en el siglo XXI (145-171). Tafalla: Ed. Txalaparta.

González, M. P. (2018). La enseñanza de la historia en el siglo XXI: saberes y prácticas. Los Polvorines: Ediciones Universidad Nacional de General Sarmiento

González, M. P. (2014a). La Historia Reciente en la escuela. Saberes y prácticas docentes en torno a la última dictadura. Buenos Aires: Universidad Nacional de General Sarmiento.

González-Valencia, G. A., Santisteban-Fernández, A., \& Pagès-Blanch, J. (2020). Finalidades de la enseñanza de la historia en futuros profesores. magis, Revista Internacional de Investigación en Educación, 13, 1-23.

Jara, M. A. (2012). Representaciones y enseñanza de la historia reciente-presente. Estudio de casos de estudiantes en formación inicial y en su primer año de docencia. Revista Enseñanza de las Ciencias Sociales, 11, 25-29.

Jara, M. A. (2017). Formar para desarrollar el pensamiento didáctico de la historia. En V. Salto (Comp.), Prácticas docentes de la enseñanza de la historia: Narrativas de experiencias (pp. 43-57). Cipolletti: Ed. UNCo.

Jara, M. A., \& Santisteban, A. (2010). Las representaciones de los estudiantes sobre la temporalidad y la HRP en la formación inicial del profesorado de historia. Aportes de una investigación. Reseñas de Enseñanza de la Historia, 8, 71-105. 
Jara, M. A., Salto, V., \& Értola, F. (2016a). Representaciones y perspectivas prácticas sobre las finalidades de la enseñanza de la historia en los/as estudiantes del profesorado. En Clio \& Asociados, 23, 69-78. Santa Fe.

Jara, M. A., Salto, V., \& Értola, F. (2016b). Representaciones y perspectivas prácticas sobre la didáctica de la historia de los/las estudiantes del profesorado. Reseñas de Enseñanza de la Historia, 14, 61-79.

Jodelet, D. (1986). La representación social: Fenómenos, concepto y teoría. En S. Moscovici (Comp.), Psicología Social I (pp. 469-494). Barcelona: Paidós.

López Facal, R. (2000). "Pensar históricamente. Una reflexión crítica sobre la enseñanza de la historia”, en Revista Íber [Versió electrònica].

Moscovici, S. (1979). La representación social, un concepto perdido. En S. Moscovici (Ed.), El Psicoanálisis, su imagen y su público. Buenos Aires: Ed. Huemul.

Pagès i Blanch, J. (2019). Lliçó magistral, Joan Pagès i Blanch Els reptes de l'ensenyament de les ciències socials, la geografia i la historia. Quin professorat, quina ciutadania, quin futur? En M. Ballbé, N. González-Monfort \& A. Santisteban (Eds.), Quin professorat, quina ciutadania, quin futur? Els reptes de l'ensenyament de les ciències socials, la geografia i la historia. Edició: GREDICS (Grup de Recerca en Didàctica de les Ciències Socials). Universitat Autònoma de Barcelona. http://rdi.uncoma.edu.ar// handle/123456789/15627

Pagès, J. (2007). La educación para la ciudadanía y la enseñanza de la Historia: cuando el futuro es la finalidad de la enseñanza del pasado. En R. M. Ávila, R. López, \& E. Fernández (Eds.), Las competencias profesionales para la enseñanza-aprendizaje de las Ciencias Sociales ante el reto europeo y la globalización (pp. 205-215). Bilbao: Asociación Universitaria del Profesorado de Didáctica de las Ciencias Sociales.

Pagés, J. (1994). La didáctica de las ciencias sociales, el currículum y la formación del profesorado. En Rev. Signos, teoría y práctica de la educación, Oct.-Dic.

Pagés, J. (1996). Las representaciones de los estudiantes de maestro sobre las Ciencias Sociales: ¿cuáles son?, ¿cómo aprovecharlas? Investigación en la Escuela, 28, 103-114.

Pagés, J. (1997). Una aproximación a la investigación sobre la formación inicial del profesorado para enseñar ciencias sociales. En La formación del profesorado y la Didáctica de las Ciencias Sociales. Sevilla: Díada editora.

Pagés, J. (2009). El desarrollo del pensamiento histórico como requisito para la formación democrática de la ciudadanía. Reseñas de la Enseñanza de la Historia, Apehun, Córdoba, Ed. Alejandría, Oct. № 7. (pp. 67-91).

Santisteban, A. (2011). Las finalidades de la enseñanza de las Ciencias Sociales. En A. Santisteban-Fernández \& J. Pagès (Coords.), Didáctica del Conocimiento del medio Social y Cultural en la Educación Primaria (pp. 63-84). Madrid: Síntesis.

Stake, R. E. (2005). Investigación con estudio de casos. Madrid, España: Morata.

Strauss, A., \& Corbin, J. (2002). Bases de la investigación cualitativa. Técnicas y procedimientos para desarrollar la teoría fundamentada. Colombia: Editorial Universidad de Antioquia.

Thorton, S. (1994). Perspectives on reflective practice in social studies education. Reflective practice in social studies. National Council for the Social Studies, 88, 5-11. 
Toledo Jofré, M. (2015). Enseñanza de 'temas controversiales' en la asignatura de historia y ciencias sociales desde la perspectiva de los profesores. Revista Estudios Pedagógicos, XLI(1), 275-292.

Van Hover, S., \& Yeager, E. (2007). I want to use my subject matter to...: The role of purpose in one U.S. secondary history teacher's instructional decision making. Canadian Journal of Education, 30(3), 670-690. 